\title{
Homogenization of the Stokes Equations with General Random Coefficients
}

\author{
Steve Wright
}

\begin{abstract}
When an attempt is made to model fluid flow in a porous medium, one is often lead to the homogenization problem for the Stokes system. One considers for each value of a small positive parameter $\varepsilon$ the solution $\left(u_{\varepsilon}, p_{\varepsilon}\right)$ of a Stokes system with coefficients and boundary conditions depending randomly on $\varepsilon$, and one seeks to prove that $\left(u_{\varepsilon}, p_{\varepsilon}\right)$ converges in some sense as $\varepsilon \rightarrow 0$ to a limit, and to derive equations which this homogenized limit satisfies. In this paper, we concentrate on the special but interesting case of $\varepsilon$-independent Dirichlet boundary conditions and general random coefficients for $n$-dimensional Stokes systems, and we use the method of stochastic two-scale convergence in the mean, introduced and studied in [3], to solve in a fairly direct and elegant way both of these problems.
\end{abstract}

Keywords: Stokes system, homogenization, partial differential equations, dynamical systems AMS subject classification: Primary 35B27, secondary 35 A99

\section{Introduction}

In [3], a stochastic variant of Allaire and Nguetseng's notion of 2-scale convergence (see $[1,5])$ was defined and studied, and the method was applied to obtain direct and simple procedures for the homogenization of elliptic and parabolic partial differential equations with random coefficients. In this paper, we will illustrate further the utility of these techniques by using them to homogenize the Stokes system with Dirichlet boundary conditions and general random coefficients.

- The homogenization problem for the Stokes system is motivated by the attempt to model the phenomenon of fluid flow through a porous medium. A standard procedure for doing this is to consider a domain filled with a large number of small channels around which the fluid flows, the channel size depending on a small parameter $\varepsilon>0$, and with the distribution of the channels allowed to vary randomly as $\varepsilon \rightarrow 0$. Along with various boundary conditions and assumptions on the geometry, this gives rise to a Stokes system with coefficients and boundary conditions depending randomly on $\varepsilon$, and one seeks to determine the limiting behavior in some sense as $\varepsilon \rightarrow 0$ of the resulting $\varepsilon$-parametrized solutions together with equations which the homogenized limit satisfies. In this paper, as in much previous work on this subject, we suppose for simplicity that the boundary conditions do not depend on $\varepsilon$. For example, the classical treatise of Bensoussan, Lions, and Papanicolaou [2: Section 10] homogenizes the Stokes system

S. Wright: Oakland Univ., Dept. of Mathematical Sciences, Rochester, MI 48309-4401, USA 
under the assumption of $\varepsilon$-independent Dirichlet boundary conditions and periodic, rapidly oscillating coefficients using a variant of Tartar's energy method. All of these results are however restricted to the case of periodic coefficients, and there appears to be little work done so far on homogenization of the Stokes system with non-periodic, random coefficients.

In Section 2 of the paper before the reader, we set up a procedure that will solve this more general problem. There we describe the stochastic calculus necessary for the statement of our homogenization results, define stochastic 2 -scale convergence in the mean, by which our homogenized limit will be attained, and record for ease of reference the results from [3] that will be used to affect the homogenization. Our theorem and its proof are given in Section 3, and some remarks are also made there concerning what more can be said when the underlying dynamics of the randomization is assumed to be ergodic.

\section{Preliminaries: stochastic differentiation and 2-scale convergence in the mean}

Let $(\Omega, \mathcal{M}, \mu)$ be a measure space with probability measure $\mu$. We define an $n$-dimension -al dynamical system on $\Omega$ as a family $\left\{T(x): x \in R^{n}\right\}$ of invertible maps $T(x): \Omega \rightarrow \Omega$ such that, for each $x \in R^{n}$, both $T(x)$ and $T(x)^{-1}$ are measureable and such that the following properties hold:

(a) $T(0)$ is the identity map on $\Omega$ and, for all $x_{1}, x_{2} \in R^{n}, T\left(x_{1}+x_{2}\right)=T\left(x_{1}\right) T\left(x_{2}\right)$.

(b) For each $x \in R^{n}$ and $E \in \mathcal{M}, \mu\left(T(x)^{-1}(E)\right)=\mu(E)$.

(c) The group $\left\{U(x): x \in R^{n}\right\}$ of unitary operators on $L^{2}(\Omega)=L^{2}(\Omega, \mathcal{M}, \mu)$ defined by $(U(x) f)(\omega)=f(T(x) \omega) \quad\left(x \in R^{n}, \omega \in \Omega, f \in L^{2}(\Omega)\right)$, is continuous in the sense that, for each $f \in L^{2}(\Omega), U(x) f \rightarrow f$ strongly in $L^{2}(\Omega)$ as $x \rightarrow 0$.

We now use a fixed $n$-dimensional dynamical system $T$ on $\Omega$ to define a stochastic differential calculus in $\Omega$ which comes from the individual coordinate actions arising from the unitary group $\left\{U(x): x \in R^{n}\right\}$. When each coordinate of $x=\left(x_{1}, \ldots, x_{n}\right)$ varies over $R$ with the other coordinates held equal to 0 in $U(x)$, we obtain $n$ oneparameter, strongly continuous, unitary group representations of $R$ on $L^{2}(\Omega)$ which pairwise commute. Let $D_{1}, \ldots, D_{n}$ denote the infinitesimal generators in $L^{2}(\Omega)$ of these one-parameter groups, with $\mathcal{D}_{1}, \ldots \mathcal{D}_{n}$ denoting their respective domains in $L^{2}(\Omega)$, i.e., for $f \in \mathcal{D}_{i}$

$$
\left(D_{i} f\right)(\omega)=\left.\frac{\partial}{\partial x_{i}}(U(x) f)(\omega)\right|_{x=0} \quad(\omega \in \Omega ; i=1, \ldots, n)
$$

where the derivative is the Frechet derivative in $L^{2}(\Omega)$. Thus $\mathcal{D}_{i}$ consists of those $f \in L^{2}(\Omega)$ for which the derivative in (2.1) converges strongly in $L^{2}(\Omega)$. Since these oneparameter groups are unitary and pairwise commute, it follows that $\sqrt{-1} D_{1}, \ldots, \sqrt{-1} D_{n}$ are closed, densely defined, self-adjoint operators on their respective domains which pairwise commute on $\mathcal{D}(\Omega)=\bigcap_{i=1}^{n} \mathcal{D}_{i}$.

For each multi-index $\alpha=\left(\alpha_{1}, \ldots, \alpha_{n}\right)$, set $D^{\alpha}=D^{\alpha_{1}} \cdots D^{\alpha_{n}}$, and then define

$$
D^{\infty}(\Omega)=\left\{f \in L^{\infty}(\Omega): D^{\alpha} f \in L^{\infty}(\Omega) \cap \mathcal{D}(\Omega), \text { for all multi-indices } \alpha\right\} .
$$


If $f \in L^{2}(\Omega)$, we define the stochastic weak derivative $D^{\alpha} f$ of $f$ as the linear functional on $D^{\infty}(\Omega)$ defined by

$$
\left(D^{\alpha} f\right)(\varphi)=(-1)^{|\alpha|} \int_{\Omega} f D^{\alpha} \varphi d \mu \quad\left(\varphi \in D^{\infty}(\Omega)\right) .
$$

We note by the skew-adjointness of $D_{i}$ that if $f \in \mathcal{D}_{i}$, then the linear functional induced by $D_{i} f$ on $L^{2}(\Omega)$ agrees with the stochastic weak derivative $D^{\alpha} f$ on $D^{\infty}(\Omega)$ when $\alpha=\left(\delta_{1 i}, \ldots, \delta_{n i}\right)$, and we hence identify $D^{\alpha} f$ with $D_{i} f, i=1, \ldots, n$.

We next introduce a subset of $D^{\infty}(\Omega)$ that will be useful for our subsequent work. For each $\varphi \in L^{\infty}(\Omega)$ and $k \in C_{0}^{\infty}\left(R^{n}\right)$, we set

$$
(\varphi * k)(\omega)=\int_{R^{n}} \varphi(T(x) \omega) \hat{k}(x) d x \quad(\omega \in \Omega)
$$

where " denotes the Fourier transform, and we let $S_{0}$ denote the set of all such functions as $\varphi$ varies over $L^{\infty}(\Omega)$ and $k$ varies over $C_{0}^{\infty}\left(R^{n}\right)$. It is clear that $S_{0} \subseteq L^{\infty}(\Omega)$. The following lemma concerning smoothness of and approximation by elements of $S_{0}$ is a combination of Lemmas 2.1 and 2.2 of [3].

Lemma 1. The following statements hold:

(a) $S_{0} \subseteq D^{\infty}(\Omega)$ and, for any multi-index $\alpha$ and $u \in S_{0}$,

$$
D^{\alpha} u(T(x) \omega)=\frac{\partial^{|\alpha|}}{\partial x_{1}^{\alpha_{1}}, \ldots, \partial x_{n}^{\alpha_{n}}} u(T(x) \omega) \quad\left(x \in R^{n}, \omega \in \Omega\right) .
$$

(b) Suppose that $L^{2}(\Omega)$ is separable. There exists a countable subset $A \subset S_{0}$ such that if $g \in \mathcal{D}(\Omega)$, then there is a sequence $\left(g_{k}\right) \subseteq A$ for which

$$
g_{k} \rightarrow g \quad \text { and } \quad \dot{D}_{i} g_{k} \rightarrow D_{i} g \quad(i=1, \ldots, n)
$$

strongly in $L^{2}(\Omega)$.

We now describe the method of convergence that will be used in our homogenization results. Let $Q$ be a bounded domain in $R^{n}$. We say that an element $\psi \in L^{2}(Q \times \Omega)$ is admissible if the function

$$
\psi_{T}:(x, \omega) \longrightarrow \psi(x, T(x) \omega) \quad((x, \omega) \in Q \times \Omega)
$$

defines an element of $L^{2}(Q \times \Omega)$. Not every element of $L^{2}(Q \times \Omega)$ is admissible $\left(\psi_{T}\right.$ may fail to be measurable), but the set of admissible elements of $L^{2}(Q \times \Omega)$ is quite large, containing for example all functions $\psi$ which have a representative which is either uniformly bounded everywhere on $Q \times \Omega$ or for which the function $x \rightarrow \psi(x, \omega)$ is continuous on $Q$ for each $\omega \in \Omega$ (see Propositions 3.1 and 3.2 of [3]).

Definition 2. A sequence $\left(u_{e}\right)$ in $L^{2}(Q \times \Omega)$ is said to stochastically 2-scale converge in the mean to $u \in L^{2}(Q \times \Omega)$ if, for every admissible $\psi \in L^{2}(Q \times \Omega)$,

$$
\lim _{\varepsilon \rightarrow 0} \int_{Q \times \Omega} u_{\varepsilon}(x, \omega) \psi\left(x, T\left(\varepsilon^{-1} x\right) \omega\right) d x d \mu=\int_{Q \times \Omega} u(x, \omega) \psi(x, \omega) d x d \mu .
$$

The utility of stochastic 2-scale mean convergence in the homogenization of partial differential equation' stems from the following basic compactness result for bounded sequences in $L^{2}(Q \times \Omega)$. 
Theorem 3 [3: Theorem 3.4]. If $L^{2}(\Omega)$ is separable, then every bounded sequence in $L^{2}(Q \times \Omega)$ has a subsequence that stochastically 2-scale converges in the mean to an element of $L^{2}(Q \times \Omega)$.

The following theorem will be the main tool we use to homogenize the Stokes system with random coefficients. It gives useful information about the stochastic 2-scale limit point of a bounded sequence $\left(u_{\varepsilon}\right)$ in $L^{2}(Q \times \Omega)$ when certain bounds are placed on the $x$-derivatives of $u_{\varepsilon}$. To state it we need to introduce some more terminology and notation.

A function $f$ on $\Omega$ is said to be invariant for $T$ (relative to $\mu$ ) if for each $x \in R^{n}$, $f \circ T(x)=f, \mu$-a.e. on $\Omega$. We will denote by $I^{2}(\Omega)$ the set of functions in $L^{2}(\Omega)$ which are invariant for $T$. It is easy to see that $I^{2}(\Omega)$ is a norm-closed, linear subspace of $L^{2}(\Omega)$. We let $P_{I^{2}}$ denote the orthogonal projection of $L^{2}(\Omega)$ onto $I^{2}(\Omega)$, and we set $M^{2}(\Omega)$ equal to the orthogonal complement of $I^{2}(\Omega)$ in $L^{2}(\Omega)$. Finally, for $f$. $\epsilon$ $L^{2}(\Omega)$ (respectively, $\left.v=\left(v_{1}, \ldots, v_{n}\right) \in L^{2}(\Omega)^{n}\right)$, we define the stochastic gradient $\nabla_{\omega} f$, divergence $\operatorname{div}_{\omega} v$, and $c u r l \operatorname{curl}_{\omega} v$ by

$$
\begin{aligned}
\nabla_{\omega} f & =\left(D_{i} f, \ldots, D_{n} f\right) \\
\operatorname{div}_{\omega} v & =\Sigma_{i} D_{i} v_{i} \\
\operatorname{curl}_{\omega} v & =\left(D_{i} v_{j}-D_{j} v_{i}\right)
\end{aligned}
$$

where $D_{i}$ is the stochastic weak derivative as defined by $(2.2)$ with $\alpha=\left(\delta_{1 i}, \ldots, \delta_{n i}\right)$, $i=1, \ldots, n$. If $w \in L^{2}(\Omega)^{n^{2}}$, with row vectors $w_{1}, \ldots, w_{n}$, we set

$$
\begin{aligned}
\operatorname{div}_{\omega} w & =\left(\operatorname{div}_{\omega} w_{1}, \ldots, \operatorname{div}_{\omega} w_{n}\right) \\
\operatorname{curl}_{\omega} w & =\left(\operatorname{curl}_{\omega} w_{1}, \ldots, \operatorname{curl}_{\omega} w_{n}\right)
\end{aligned}
$$

The proof of the next theorem can be obtained by a straightforward modification of the proof of Theorem 3.7/(b) of [3].

Theorem 4. Assume that $L^{2}(\Omega)$ is separable, and let $X$ be a norm-closed, convex subset of $H^{1}(Q)^{n}$. Suppose that $\left(u_{\varepsilon}\right)$ is a sequence in $L^{2}(Q \times \Omega)^{n}$ which for $\varepsilon>0$ satisfies the following conditions:

(i) $u_{\varepsilon}(\cdot, \omega) \in X$ for $\mu$-a.e. $\omega \in \Omega$.

(ii) $\int_{\Omega}\left\|u_{\epsilon}(\cdot, \omega)\right\|_{H^{1}(Q)^{n}}^{2} d \mu \leq C$ for some absolute constant $C>0$.

Then there exists elements $u \in H^{1}\left(Q, L^{2}(\Omega)\right)^{n}$ and $v \in L^{2}(Q \times \Omega)^{n^{2}}$ and a subsequence $\left(u_{\varepsilon}\right)$ which satisfy the following conditions:

(iii) For a.e. $x \in Q, u(x, \cdot) \in I^{2}(\Omega)^{n}$ and for $\mu$-a.e. $\omega \in \Omega, u(\cdot, \omega) \in X$.

(iv) For a.e. $x \in Q,\left(\nabla_{x} u\right)(x, \cdot) \in I^{2}(\Omega)^{n^{2}}$.

(v) For a.e. $x \in Q, v(x, \cdot) \in M^{2}(\Omega)^{n^{2}}$ and $\operatorname{curl}_{\omega} v(x, \cdot)=0$.

(vi) $\left(u_{\varepsilon}\right)$ and $\left(\nabla_{x} u_{\varepsilon}\right)$ stochastically 2-scale converges in the mean to $u$ and $v+\nabla_{x} u$, respectively.

The final tool that we will need is the following stochastic analog of the "div-curl" lemma from the theory of compensated compactness. To state it, we recall that if $A$ and $B$ are $(n \times n)$-matrices, then $A: B=$ Trace $A^{T} B$ denotes their standard inner product. 
Lemma 5. Suppose $u, v \in L^{2}(\Omega)^{n^{2}}$ satisfy $\operatorname{div}_{\omega} u=0$ and $\operatorname{curl}_{\omega} v=0$. Then

$$
\int_{\Omega} u: v d \mu=\int_{\Omega} P_{I^{2}} u: P_{I^{2}} v d \mu
$$

Proof. If $\operatorname{div}_{\omega} u=0$ and $\operatorname{curl}_{\omega} v=0$; then $\operatorname{div}_{\omega} u_{i}=0$ and $\operatorname{curl}_{\omega} v_{i}=0$, where $u_{i}$ and $\left.v_{i}\right)$ is the $i$-th row of $u$ and $v$, respectively $(i=1, \ldots, n)$. Hence by Lemma 2.4 of $[3]$,

$$
\int_{\Omega} u: v d \mu=\sum_{i} \int_{\Omega} u_{i} \cdot v_{i} d \mu=\sum_{i} \int_{\Omega} P_{I^{2} u_{i}} \cdot P_{I^{2} v_{i}} d \mu=\int_{\Omega} P_{I^{2} u}: P_{I^{2}} v d \mu
$$

and the statement is true

In order to state our homogenization results precisely, we need to describe a dynamical system acting on $Q \times \Omega$ that arises naturally from $T$. Define for each $y \in R^{n}$ the map $\widetilde{T}(y)=\mathrm{id}_{Q} \times T: Q \times \Omega \rightarrow Q \times \Omega$ by

$$
\widetilde{T}(y)(x, \omega)=(x, T(y) \omega) \quad((x, \omega) \in Q \times \Omega) .
$$

It then follows straightforwardly that $\left\{\widetilde{T}(y): y \in R^{n}\right\}$ defines an $n$-dimensional dynamical system on $Q \times \Omega$ with invariant measure given by the product of Lebesgue measure on $Q$ and $\mu$. We denote by $I^{2}(Q \times \Omega)$ the subspace of $L^{2}(Q \times \Omega)$ consisting of the functions that are invariant for $\tilde{T}$ relative to $d x \times d \mu$, and we denote by $\tilde{P}$ the orthogonal projection of $L^{2}(Q \times \Omega)$ onto $I^{2}(Q \times \Omega)$. It now follows from the ergodic theorem [4: Theorem VIII.7.10 and Corollary VIII.7.2] that, for $u=\Sigma_{i} f_{i} g_{i} \in L^{2}(Q) \otimes L^{2}(\Omega)$ and for $(d x \times d \mu)$-a.e. $(x, \omega) \in Q \times \Omega$,

$$
\begin{aligned}
\tilde{P}(u)(x, \omega) & =\lim _{t \rightarrow+\infty} \frac{1}{t^{n}} \int_{[0, t]^{n}} u(x, T(y) \omega) d y \\
& =\sum_{i} f_{i}(x) \lim _{t \rightarrow+\infty} \frac{1}{t^{n}} \int_{[0, t]^{n}} g_{i}(T(y) \omega) d y \\
& =\sum_{i} f_{i}(x)\left(P_{I^{2}} g\right)(\omega) \\
& =P_{I^{2}}[u(x, \cdot)](\omega)
\end{aligned}
$$

and consequently from this and the strong density of $L^{2}(Q) \otimes L^{2}(\Omega)$ in $L^{2}(Q \times \Omega)$, we have

$$
\tilde{P}(f g)=f \tilde{P}(g) \text { for all } f \in L^{\infty}(Q), g \in L^{2}(Q \times \Omega) \text {. }
$$

Equations (2.3) and (2.4) will be needed in the proof of Theorem 6 in the next section. 


\section{Homogenization of the Stokes system with random coefficients}

Let $\Omega$ and $T$ be as in Section 2 , with $L^{2}(\Omega)$ assumed separable. We suppose for simplicity that the bounded domain $Q \subset R^{n}$ has a $C^{2}$ boundary $\Gamma$, and let $g \in L^{2}(Q)$ and $u_{0} \in H^{1 / 2}(\Gamma)^{n}$ satisfy the compatibility condition

$$
\int_{Q} g d x=\int_{\Gamma} u_{0} \cdot n d \Gamma
$$

where $n$ is the outward unit normal to $\Gamma$. Consider for $\varepsilon>0$ and $f \in L^{2}(Q)$ the Stokes system

$$
\begin{aligned}
& \nabla p_{\varepsilon}-\operatorname{div}_{x}\left\{\mathbf{A}\left(x, T\left(\varepsilon^{-1} x\right) \omega\right) \nabla u_{\varepsilon}\right\}=f \quad \text { in } Q \\
& \operatorname{div} u_{\varepsilon}=g \text { in } Q \\
& u_{\varepsilon}=u_{0} \text { on } \Gamma
\end{aligned}
$$

where $u_{\varepsilon}=\left(u_{1 \varepsilon}, \ldots, u_{n \varepsilon}\right)$ represents the velocity, $p_{\varepsilon}$ represents the pressure, and $\mathbf{A}$ is a strongly elliptic, symmetric, fourth-order viscosity tensor defined on $Q \times \Omega$, i.e., $\mathbf{A}$ satisfies

$$
\mathbf{A}_{i j h k}=\mathbf{A}_{j i h k}=\mathbf{A}_{h k i j}
$$

and there exists constants $0<\alpha \leq \beta$ such that, for $(d x \times d \mu)$-a.e. $(x, \omega) \in Q \times \Omega$,

$$
\alpha|\xi|^{2} \leq \sum \mathbf{A}_{i j h k}(x, \omega) \xi_{i j} \xi_{h k} \leq \beta|\xi|^{2}
$$

-We also suppose that $\mathbf{A}_{i j h k}$ is $(d x \times d \mu)$-essentially bounded on $Q \times \Omega$ and is an admissible element of $L^{2}(Q \times \Omega)$ for each $i, j, h$, and $k$. We emphasize that these smoothness conditions on the coefficients are very general. Given the assumption of strong ellipticity, the admissibility condition on $\mathbf{A}_{i j k l}$ means only that $(x, \omega) \rightarrow \mathbf{A}_{i j k l}(x, T(x) \omega)$ is measurable on $Q \times \Omega$, and this is close to being the weakest smoothness assumption that one can require in order to guarantee the existence of weak solutions to system (3.1) - (3.3) for $\omega \in \Omega$ (cf. [6, 9 - 11], which consider homogenization problems with random coeffcients that are required to satisfy much stronger conditions). It is hence a consequence of standard existence and uniqueness theory for Stokes systems (see [8]: Chapter 1] that system (3.1) - (3.3) has a unique weak solution $\left(u_{\varepsilon}(\cdot, \omega), p_{\varepsilon}(\cdot, \omega)\right) \in H^{1}(Q)^{n} \times L_{0}^{2}(Q)$ for $\varepsilon>0$ and $\omega \in \Omega$ (here $L_{0}^{2}(Q)$ denotes those $f \in L^{2}(Q)$ with $\int_{Q} f d x=0$ ). We are interested in the limiting behavior of the sequences $\left(u_{\varepsilon}\right)$ and $\left(p_{\varepsilon}\right)$ as $\varepsilon \rightarrow 0$, and to this end, we will prove the following with

Theorem 6. There exists $u \in H^{1}\left(Q, I^{2}(\Omega)\right)^{n}, p \in L^{2}(Q \times \Omega)$, and $\xi \in L^{2}(Q \times \Omega)^{n^{2}}$

$$
\begin{aligned}
& \int_{Q} p(x, \cdot) d x \in M^{2}(\Omega) \\
& \text { Trace } \xi(x, \omega)=0 \quad \text { for }(d x \times d \mu)-\text { a.e. }(x, \omega) \in Q \times \Omega \\
& \operatorname{curl}_{\omega} \xi(x, \cdot)=0, P_{I^{2}} \xi(x, \cdot)=0 \text { for a.e. } x \in Q
\end{aligned}
$$


such that the sequence $\left(u_{e}, \nabla_{z} u_{e}, p_{e}\right)$ stochastically 2-scale converges in the mean as $\varepsilon \rightarrow 0$ to $\left(u, \xi+\nabla_{x} u, p\right)$ and $(u, \xi, p)$ is the unique weak solution to system (9.4) - (9.6) and

$$
\begin{aligned}
& -\operatorname{div}_{\omega}\left\{\mathbf{A}\left[\nabla_{x}(u+\xi](x, \cdot)\right\}+\nabla_{\omega} p(x, \cdot)=0 \quad \text { for a.e. } x \in Q\right. \\
& -\operatorname{div}_{x}\left\{\tilde{P}\left(\mathbf{A}\left[\nabla_{x} u+\xi\right]\right)(\cdot, \omega)\right\}+\nabla_{x} \tilde{P}(p)(\cdot, \omega)=f \text { in } Q, \text { for } \mu-\text { a.e.w } \in \Omega \\
& \operatorname{div}_{x} u(\cdot, \omega)=g \text { in } Q, \text { for } \mu-\text { a.e.w } \in \Omega \\
& u(\cdot, \omega)=u_{0} \text { on } \Gamma, \text { for } \mu-\text { a.e. } \omega \in \Omega \text {. }
\end{aligned}
$$

Proof. One easily checks via the standard estimates that $(x, \omega) \rightarrow u_{\varepsilon}(x, \omega)$ and $(x, \omega) \rightarrow p_{\varepsilon}(x, \omega)$ are measurable functions on $Q \times \Omega$ for each $\varepsilon>0$, and that there is a constant $C>0$ such that, for $\varepsilon>0$ and $\mu$-a.e. $\omega \in \Omega$,

$$
\left\|u_{\varepsilon}(\cdot, \omega)\right\|_{H^{1}(Q)^{n}} \leq C \quad \text { and } \quad\left\|p_{e}(\cdot, \omega)\right\|_{L^{2}(Q)} \leq C
$$

whence

$$
\left\|u_{c}\right\|_{H^{1}\left(Q, L^{2}(\Omega)\right)^{n}} \leq C \quad \text { and } \quad\left\|p_{\varepsilon}\right\|_{L^{2}(Q \times \Omega)} \leq C .
$$

Thus by Theorems 3 and 4, with $X$ the set of all elements in $H^{1}(Q)^{n}$ with divergence $g$ and boundary values $u_{0}$, to each subsequence $\left(u_{\varepsilon}\right)$ and $\left(p_{\varepsilon}\right)$, there corresponds elements $u \in H^{1}\left(Q, I^{2}(\Omega)\right)^{n}, p \in L^{2}(Q \times \Omega), \xi \in L^{2}(Q \times \Omega)^{n^{2}}$, and subsequences $\left(u_{e}\right)$ and $\left(p_{e}\right)$ such that

$$
\begin{aligned}
& \operatorname{div}_{x} u(\cdot, \omega)=g \text { in } Q \text { and } u(\cdot, \omega)=u_{0} \text { on } \Gamma \text { for } \mu-\text { a.e. } \omega \in \Omega \\
& \operatorname{curl}_{\omega} \xi(x, \cdot)=0 \text { and } P_{I^{2}} \xi(x, \cdot)=0 \text { for a.e. } x \in Q
\end{aligned}
$$

and, respectively,

$$
\left(u_{\varepsilon}\right),\left(\nabla_{x} u_{\varepsilon}\right),\left(p_{\varepsilon}\right) \text { stoch. } 2 \text {-scale conv. in the mean to } u, \xi+\nabla_{x} u, p \text {. }
$$

In order to prove that $(u, \xi, p)$ satisfies (3.4) - (3.10), we observe first that (3.6), (3.9), and (3.10) follow from (3.11) and (3.12). We next let $A$ denote the approximating subset from Lemma $1 /(\mathrm{b})$ and choose $\varphi \in\left[C_{0}^{\infty}(Q) \otimes I^{2}(\Omega)\right]^{n}, h \in C_{0}^{\infty}(Q)$, and $k \in A^{n}$. The weak form of equation (3.1) hence implies for $\varepsilon>0$ that

$$
\begin{aligned}
\int_{Q \times \Omega} \mathbf{A}(x, & \left.T\left(\varepsilon^{-1} x\right) \omega\right) \nabla_{x}\left(u_{e}(x, \omega)\right) \\
& :\left[\nabla_{x}(\varphi(x, \omega))+\varepsilon \nabla_{x}\left(h(x) k\left(T\left(\varepsilon^{-1} x\right) \omega\right)\right)\right] d x d \mu \\
= & \int_{Q \times \Omega} f(x) \cdot\left(\varphi(x, \omega)+\varepsilon h(x) k\left(T\left(\varepsilon^{-1} x\right) \omega\right)\right) d x d \mu \\
& +\int_{Q \times \Omega} p_{e}(x, \omega)\left[\operatorname{div}_{x} \varphi(x, \omega)+\varepsilon \operatorname{div}_{x}\left(h(x) k\left(T\left(\varepsilon^{-1} x\right) \omega\right)\right)\right] d x d \mu .
\end{aligned}
$$

Observe next that by Lemma $1 /(\mathrm{a})$, for $\varepsilon>0$,

$$
\varepsilon \operatorname{div}_{x}\left(h(x) k\left(T\left(\varepsilon^{-1} x\right) \omega\right)\right)=\varepsilon \nabla h(x) \cdot k\left(T\left(\varepsilon^{-1} x\right) \omega\right)+h(x) \operatorname{div}_{\omega}\left(k\left(T\left(\varepsilon^{-1} x\right) \omega\right)\right)
$$


and

$$
\varepsilon \nabla_{x}\left(h(x) k\left(T\left(\varepsilon^{-1} x\right) \omega\right)\right)=\varepsilon\left(k_{i}\left(T\left(\varepsilon^{-1} x\right) \omega\right) h_{x_{j}}(x)\right)+h(x) \nabla_{\omega}\left(k\left(T\left(\varepsilon^{-1} x\right) \omega\right)\right) .
$$

We substitute (3.15) and (3.16) into (3.14) and pass to the limit as $\varepsilon \rightarrow 0$ in the equation which results to conclude by $(3.13)$ that

$$
\begin{aligned}
\int_{Q \times \Omega} \mathbf{A}(x, \omega)\left[\nabla_{x}(u(x, \omega))+\xi(x, \omega)\right]:\left[\nabla_{x} \varphi(x, \omega)+h(x)\left(\nabla_{\omega} k\right)(\omega)\right] d x d \mu \\
=\int_{Q \times \Omega} f(x) \cdot \varphi(x, \omega) d x d \mu+\int_{Q \times \Omega} p(x, \omega) \operatorname{div}_{x} \varphi(x, \omega) d x d \mu \\
\quad+\int_{Q \times \Omega} p(x, \omega) h(x) \operatorname{div}_{\omega} k(\omega) d x d \mu .
\end{aligned}
$$

Since $\varphi, h$, and $k$ are arbitrary, it will follow from (2.4), (3.17), and Lemma $1 /(\mathrm{b})$ that $(u, \xi, p)$ is a weak solution of (3.7) and (3.8), provided we verify that integration over $Q$ intertwines $\tilde{P}$ and $P_{I^{2}}$, i.e., for each $g \in L^{2}(Q \times \Omega)$,

$$
P_{I^{2}}\left(\int_{Q} g(x, \cdot) d x\right)=\int_{Q} \tilde{P}(g)(x, \cdot) d x .
$$

But this follows from the fact that the mappings

$$
g \rightarrow P_{I^{2}}\left(\int_{Q} g(x, \cdot) d x\right) \quad \text { and } \quad g \rightarrow \int_{Q} \tilde{P}(g)(x, \cdot) d x \quad\left(g \in L^{2}(Q \times \Omega)\right)
$$

define bounded linear operations from $L^{2}(Q \times \Omega)$ into $L^{2}(\Omega)$ which agree by virtue of (2.3) on the strongly dense subset $L^{2}(Q) \otimes L^{2}(\Omega)$ of $L^{2}(Q \times \Omega)$.

To verify (3.4) we let $\psi \in I^{2}(\Omega)$ and deduce from (3.13) and the definition of stochastic 2 -scale mean convergence that

$$
f_{e}=\int_{\Omega} p_{e}(\cdot, \omega) \psi(\omega) d \mu \quad \longrightarrow \quad f=\int_{\Omega} p(\cdot, \omega) \psi(\omega) d \mu
$$

weakly in $L^{2}(Q)$ (cf. [3: Proposition 3.5/(b)]). Since for $\varepsilon>0, f_{\varepsilon} \in L_{0}^{2}(Q)$, it follows that $f \in L_{0}^{2}(Q)$, i.e.,

$$
\int_{\Omega} \psi(\omega)\left(\int_{Q} p(x, \omega) d x\right) d \mu \doteq 0
$$

Since $\psi \in I^{2}(\Omega)$ is arbitrary, this shows that $p$ satisfies (3.4).

It remains only to prove that $\xi$ has zero trace. To see this, we write the equation $\operatorname{div}_{x} u_{e}=g$ in the form $I: \nabla_{x} u_{e}=g$, where $I$ is the $(n \times n)$-matrix $\left(\delta_{i j}\right)$, and deduce from (3.13) and the $\mu$-invariance of $T$ that $g=I:\left(\xi+\nabla_{z} u\right)$, and so by (3.11),

$$
\text { Trace } \xi=\sum_{i} \xi_{i i}=0 \quad \text { on } Q \times \Omega \text {. }
$$


We now show that $(u, \xi, p)$ is uniquely determined by (3.4) - (3.10): This will imply that $u, \xi$, and $p$ are independent of the subsequences arising in the previous portion of the argument, and the theorem will hence be established. Suppose then that $\left(u_{1}, \xi_{1}, p_{1}\right)$ and $\left(u_{2}, \xi_{2}, p_{2}\right)$ are solutions of (3.4) - (3.10). If we now set $u=u_{1}-u_{2}, \xi=\xi_{1}-\xi_{2}$ and $p=p_{1}-p_{2}$, then $(u, \xi, p)$ satisfies $\left(3.4-(3.10)\right.$ with $g=0, u_{0}=0$ and $f=0$, and so we must prove that $u=0, \xi=0$ and $p=0$.

To this end, let

$$
\zeta(x, \omega)=\nabla_{x} u(x, \omega)+\xi(x, \omega) \quad((x, \omega) \in Q \times \Omega)
$$

Recall now from Theorem $4 /\left(\right.$ iv) that $\nabla_{x} u(x, \cdot) \in I^{2}(\Omega)^{n^{2}}$ for a.e. $x \in Q$, and this together with (3.12) implies that, for a.e. $x \in Q$,

$$
\begin{aligned}
\operatorname{curl}_{\omega}\left[\zeta(x, \cdot)-\nabla_{x} u(x, \cdot)\right] & =0 \\
P_{I^{2}}(\zeta(x, \cdot)) & =\nabla_{x} u(x, \cdot) .
\end{aligned}
$$

Set

$$
g(x, \omega)=\mathbf{A}(x, \omega) \zeta(x, \omega)-p(x, \omega) I \quad((x, \omega) \in Q \times \Omega) .
$$

Then $g \in L^{2}(Q \times \Omega)^{n^{2}}$ and, from (3.7),

$$
\operatorname{div}_{\omega} g(x, \cdot)=0 \quad \text { for a.e. } x \in Q .
$$

By (3.18) - (3.20) and Lemma 5, for a.e. $x \in Q$,

$$
\begin{aligned}
\int_{\Omega}[\zeta(x, \omega) & \left.-\nabla_{x} u(x, \omega)\right]: g(x, \omega) d \mu \\
= & \int_{\Omega}\left[P_{I^{2}}(\zeta(x, \omega))-\nabla_{x} u(x, \omega)\right]: P_{I^{2}} g(x, \omega) d \mu \\
& =0
\end{aligned}
$$

i.e.,

$$
\begin{aligned}
\int_{\Omega} \mathbf{A}(x, \omega) \zeta(x, \omega): \zeta(x, \omega)-\int_{\Omega} \zeta(x, \omega): p(x, \omega) I d \mu \\
=\int_{\Omega}(\mathbf{A}(x, \omega) \zeta(x, \omega)-p(x, \omega) I): \nabla_{x} u(x, \omega) d \mu
\end{aligned}
$$

Observe next that since $u \in H_{0}^{1}\left(Q, I^{2}(\Omega)\right)^{n}$, we may approximate $u \in H^{1}\left(Q, L^{2}(\Omega)\right)^{n}$ by elements of $\left(C_{0}^{\infty}(Q) \otimes I^{2}(\Omega)\right)^{n}$, and so by (3.17), with $k=0$ and $f=0$, we obtain

$$
\begin{aligned}
& \int_{Q \times \Omega}(\mathbf{A}(x, \omega) \zeta(x, \omega)-p(x, \omega) I): \nabla_{x} u(x, \omega) d x d \mu \\
&= \int_{Q \times \Omega} \mathbf{A}(x, \omega) \zeta(x, \omega): \nabla_{x} u(x, \omega) d x d \mu \\
&-\int_{Q \times \Omega} p(x, \omega) \operatorname{div}_{x} u(x, \omega) d x d \mu \\
&= 0
\end{aligned}
$$


Integrating (3.21) over $Q$ and using (3.5), (3.9) with $g=0$, and (3.22) in the equation which results yields

$$
\begin{aligned}
\int_{Q \times \Omega} \mathbf{A} & (x, \omega) \zeta(x, \omega): \zeta(x, \omega) d x d \mu \\
& =\int_{Q \times \Omega} \zeta(x, \omega): p(x, \omega) I d x d \mu \\
& =\int_{Q \times \Omega} \nabla_{x} u(x, \omega): p(x, \omega) I d x d \mu+\int_{Q \times \Omega} \xi(x, \omega): p(x, \omega) I d x d \mu \\
& =\int_{Q \times \Omega} p(x, \omega) \operatorname{div}_{x} u(x, \omega) d x d \mu+\int_{Q \times \Omega} p(x, \omega) \text { Trace } \xi(x, \omega) d x d \mu \\
& =0 .
\end{aligned}
$$

The strong ellipticity of $\mathbf{A}$ and (3.23) produces $\zeta=0$ on $Q \times \Omega$, whence by (3.19), $\nabla_{x} u$, and hence $u$, is 0 on $Q \times \Omega$. But then by the definition of $\zeta, \xi=0$ on $Q \times \Omega$. This in turn implies by (3.7) that

$$
\nabla_{\omega} p(x, \cdot)=0 \quad \text { for a.e. } x \in Q
$$

which implies by Lemma $2.3 /(\mathrm{a})$ of [3] that $p \in L^{2}\left(Q, I^{2}(\Omega)\right)$. But we deduce now from (2.3) that

$$
\text { range of } \tilde{P}=L^{2}(Q \times \Omega) \text {-norm closure of } L^{2}(Q) \otimes I^{2}(\Omega)=L^{2}\left(Q, I^{2}(\Omega)\right)
$$

and this coupled with (3.8) hence yields

$$
\nabla_{x} p(\cdot, \omega)=\nabla_{x} \tilde{P}(p)(\cdot, \omega)=0 \quad \text { for } \mu-\text { a.e. } \omega \in \Omega \text {. }
$$

It follows that $p=p(\omega)$ is a function of $\omega$ only and $p \in I^{2}(\Omega)$. But by (3.4), we also have $p \in M^{2}(\Omega)$, and since $I^{2}(\Omega)$ and $M^{2}(\Omega)$ are orthogonal in $L^{2}(\Omega)$, this gives $p=0$

Remark. We now suppose that the dynamical system $T$ is ergodic, i.e., whenever $E$ is a $\mu$-measurable subset of $\Omega$ for which $T(x)(E)=E$ for all $x \in R^{n}$, then $\mu(E)$ is either 0 or 1 . It then follows that $I^{2}(\Omega)$ consists only of functions that are constant $\mu$-a.e. on $\Omega$, and so the homogenized limit $u$ of Theorem 6 is in fact a function of $x$ only.

If 'we set.

$$
\mathbf{A}^{*}(x)=\int_{\Omega} \dot{\mathbf{A}}(x, \omega) d \mu \quad \text { and } \quad p^{*}(x)=\int_{\Omega} p(x, \omega) d \mu \quad(x \in Q),
$$

then $p^{*} \in L^{2}(Q)$ and $\mathbf{A}^{*}$ is a symmetric, strongly elliptic, fourth-order tensor on $Q$. By using the fact that when $T$ is ergodic,

$$
P_{I^{2} g}=\int_{\Omega} g d \mu \quad \text { for all } g \in L^{2}(\Omega)
$$


it follows from (2.3) and the strong density of $L^{2}(Q) \otimes L^{2}(\Omega)$ in $L^{2}(Q \times \Omega)$ that, for $h \in L^{2}(Q \times \Omega)$,

$$
\tilde{P}(h)(x, \omega)=\int_{\Omega} h(x, \eta) d \mu(\eta) \quad \text { for }(d x \times d \mu)-\text { a.e. }(x, \omega) \in Q \times \Omega .
$$

We can thus write system $(3.8)-(3.10)$ in the form

$$
\begin{aligned}
-\operatorname{div}\left(\mathbf{A}^{*} \nabla u\right)+\nabla p^{*} & =f+\operatorname{div} \int_{\mathbf{\Omega}} \mathbf{A}(\cdot, \omega) \xi(\cdot, \omega) d \mu \text { in } Q \\
\operatorname{div} u & =g \text { in } Q \\
u & =u_{0} \text { on } \Gamma
\end{aligned}
$$

Since the term on the right-hand side of (3.24) can be interpreted as an element of $H^{-1}(Q)^{n}$, this exhibits $\left(u, p^{*}\right)$ as the solution of a Stokes system on $Q$.

Allaire [1] takes notice of a similar phenomenon that occurs when he homogenizes via the techniques of [7] a Stokes system which models flow in certain bubbly fluids. He treats the case of periodic, rapidly oscillating coefficients, an assumption which requires that the size, shape, and arrangement of the bubbles be kept fixed, and which hence makes the model unrealistic. Unfortunately, as Allaire observes himself, this assumption also makes the Stokes system for the homogenized limit and the Stokes system for the velocity in the microstructure have the same forcing term, and so no significant effects are detected by the homogenized equations. Equation (3.24) suggests that if the bubbles are allowed to propogate randomly, a much more realistic assumption, then our homogenization procedure will capture effects present in the macrostructure, with the external forces there influenced explicitly by the vector field $\xi$.

Acknowledgement. I wish to thank Andro Mikelic' for several very helpful conversations concerning the contents and methods of this paper.

\section{References}

[1] Allaire, G.: Homogenization and two-scale convergence. SIAM J. Math. Anal. 23 (1992), 1482 - 1518.

[2] Bensoussan, A., Lions, J. L. and G. Papanicolaou: Asymptotic Analysis for Periodic Structures. Amsterdam: North-Holland Publ. Comp. 1978.

[3] Bourgeat, A., Mikelić, A. and S. Wright: Stochastic two-scale convergence in the mean and applications. J. Reine Angew. Math. (to appear).

[4] Dunford, N. and J. T. Schwartz: Linear Operators. Part I: General Theory. New York: John Wiley \& Sons 1963.

[5] Nguetseng, G.: A general convergence result for a functional related to the theory of homegenization. SIAM J. Math. Anal. 20 (1986), 608 - 623.

[6] Papanicolaou, G. S. and S. R. S. Varadhan: Boundary value problems with rapidly oscillating coefficients. In: Proceedings of a colloquium on random fields (eds: J. Fritz, J. L. Lebowitz and D. Szasz). Amsterdam: North-Holland Publ. Comp. 1979, pp. 835 - 873. 
[7] Tartar, L.: Convergence of the homogenization process. In: Nonhomogeneous media and vibration theory (Lect. Notes Phys.: Vol. 127; ed.: E. Sanchez-Palencia). Berlin Heidelberg - New York: Springer-Verlag 1980, Appendix.

[8] Temam, R.: Navier-Stokes Equations. Amsterdam: North-Holland Publ. Comp. 1984.

[9] Zhikov, V. V., Koslov, S. M., Oleinik, O. A. and K. T. Ngoan: Averaging and G.Convergence of Differential Operators. Russian Math. Surveys 34 (1979), $69-147$.

[10] Zhikov, V. V., Koslov, S. M. and O. A. Oleinik: Averaging of parabolic operators. Trans. Moscow Math. Soc. 45 (1984), 189 - 241.

[11] Zhikov, V. V., Koslov, S. M. and O. A. Oleinik: Homogenization of Differential Operators. Berlin - Heidelberg - New York: Springer-Verlag (to appear).

Received 16.09.1993 